\title{
Sutureless repair and deep sedation for a blow-out type post-infarction left ventricular free wall rupture
}

\author{
Sho Kusadokoro ${ }^{1}$, Manabu Shiraishi ${ }^{1}$, Daijiro Hori ${ }^{1}$, and Atsushi Yamaguchi ${ }^{1}$ \\ ${ }^{1}$ Saitama Medical Center, Jichi Medical University
}

April 10, 2021

\begin{abstract}
A 71-year-old woman who was resuscitated from cardiac arrest after pericardial drainage, was admitted to our hospital. Enhanced computed tomography demonstrated pericardial effusion due to rupture of posterior ventricular myocardium. She underwent emergent surgical repair. A $20-\mathrm{mm}$ tear in the extensively necrotic left ventricular posterior wall and active hemorrhage were identified. Sutureless repair using three sheets of TachoSil (CSL Behring, Tokyo, Japan) and fibrin glue was performed. To reduce ventricular pressure to avoid re-rupture and formation of ventricular aneurysm, deep sedation was followed in the intensive care unit for 2 weeks. The patient returned to her normal daily life and is progressing well for more than 5 months after the surgery.
\end{abstract}

Sutureless repair and deep sedation for a blow-out type post-infarction left ventricular free wall rupture

Running Head: Sutureless repair of ventricle

Sho Kusadokoro, MD, Manabu Shiraishi, MD, PhD, Daijiro Hori, MD, PhD, Atsushi Yamaguchi, MD, PhD Department of Cardiovascular Surgery, Saitama Medical Center, Jichi Medical University, Saitama, Japan

Data availability statement: not applicable for this case report.

Funding: none

Conflict of interest: none

IRB approval: waived for this case report.

Patient consent: Informed consent was obtained from the patient.

Clinical trial registration: not applicable for this case report.

Address for correspondence : Manabu Shiraishi MD, $\mathrm{PhD}$.

ORCiD ID: https://orcid.org/0000-0002-4491-7473

1-847 Amanumacho, Omiya-ku, Saitama, 330-0834, Japan.

Tel: +81-48-647-2111, Fax: +81-48-645-0621, E-mail: manabu@omiya.jichi.ac.jp

\section{Abstract}

A 71-year-old woman who was resuscitated from cardiac arrest after pericardial drainage, was admitted to our hospital. Enhanced computed tomography demonstrated pericardial effusion due to rupture of posterior ventricular myocardium. She underwent emergent surgical repair. A 20-mm tear in the extensively necrotic 
left ventricular posterior wall and active hemorrhage were identified. Sutureless repair using three sheets of TachoSil (CSL Behring, Tokyo, Japan) and fibrin glue was performed. To reduce ventricular pressure to avoid re-rupture and formation of ventricular aneurysm, deep sedation was followed in the intensive care unit for 2 weeks. The patient returned to her normal daily life and is progressing well for more than 5 months after the surgery.

Key Words : Left ventricular free wall rupture; Sutureless repair; Myocardial infarction

\section{Introduction}

Post-infarction left ventricular free wall rupture (LVFWR) is a life-threatening disease, for which the reported mortality is $42.9 \%$. (1) In particular, the blow-out type is catastrophic, and immediate surgery is necessary for the patient to survive. Although several surgical methods have been reported, including suture repair and sutureless repair, the relative success rates of these surgeries are uncertain. Recently, the sutureless repair of LVFWR has been gaining in popularity, but this approach may not be sufficient for hemostasis in patients with blow-out type. (1) Furthermore, sutureless repair may be followed by re-rupture and formation of ventricular aneurysm.

Here, we report a successful treatment of blow-out LVFWR via sutureless repair and deep sedation for 2 weeks after the surgery.

\section{Case report}

A 71-year-old woman who had a history of hypertension and dyslipidemia was admitted to a hospital due to acute onset of chest and back pain. She suddenly went into cardiac arrest and cardiopulmonary resuscitation was started. Echocardiography revealed a pericardial effusion, and she underwent pericardial drainage and was successfully resuscitated. She was suspected of having a type A aortic dissection and was transferred to our hospital. Enhanced computed tomography (CT) demonstrated pericardial effusion and a lack of enhancement in the lateral to posterior ventricular wall with a suspected tear in the unenhanced myocardium. (Fig 1 and Supplemental movie) Electrocardiography demonstrated ST-T depression on the II, III, aVf, and V3-V6 leads. She was diagnosed with post-infarction LVFWR and was transferred to the operating room immediately.

During the induction of general anesthesia, the patient's blood pressure (BP) dropped to 40/30 mmHg. Median sternotomy and pericardiotomy were performed promptly, and a 1,000-ml of hematoma was drainaged. Extensive necrosis and a 20-mm tear, accompanied by active hemorrhage, were identified in the posterior wall. The rupture site was manually reduced using TachoSil (CSL Behring, Tokyo, Japan) and fibrin glue from the epicardial surface. To reduce the likelihood of further hemorrhage from the left ventricle, three sheets of TachoSil were applied over a wide area, not only at the rupture site, but also over the entire necrotic area. Hemostasis was achieved and the patient's vital signs stabilized.

After admission to the intensive care unit (ICU), the patient was deeply sedated and maintained in this state using propofol and midazolam. For 2 weeks, her systolic BP and heart rate were maintained at $<100 \mathrm{mmHg}$ and $<60$ beats/min to reduce ventricular pressure load. Serum lactate level was also monitored for evaluation of systemic perfusion. The maximum lactate level was $4.06 \mathrm{mmol} / \mathrm{L}$ on ICU admission, which recovered to a normal level without re-elevation. The average lactate during the 2 weeks period was $1.05 \pm 0.42 \mathrm{mmol} / \mathrm{L}$. The patient experienced transient acute kidney injury due to post cardiac arrest syndrome and was treated by continuous renal replacement therapy from postoperative day (POD) 7 to 20. Echocardiography was frequently performed to identify signs of re-rupture and left ventricular aneurysm, and CT was performed on PODs 6 and 13 for the same reason. Fortunately, these complications did not occur. The patient's sedation was reduced gradually from POD 14, and her state of consciousness gradually improved with full recovery after 1 month. Coronary CT demonstrated stenosis in her peripheral right coronary artery, but no sign of ventricular aneurysm (Fig 2). The patient was transferred to another hospital for rehabilitation on POD 78, and was discharged after 2 months. She is now following her normal daily routine for more than 5 months after the surgery, without experiencing ventricular aneurysm or pseudoaneurysm. 


\section{Discussion}

LVFWR is a catastrophic complication of acute myocardial infarction (AMI) that has become rare due to advancement of prompt coronary reperfusion strategies, with a reported incidence of $2 \%$ following AMI. (2) $87.5 \%$ of LVFWR is reported to occurs in the first week of AMI and $55.6 \%$ of LVFWR occurs in the first 48 hours. (3)

Matteucci et al. published a systemic review of the use of suture or sutureless repair for LVFWR, (4) in which they found that in-hospital mortality $(13.8 \%$ vs . $14 \%$, respectively) was comparable. The fragility of the myocardium following infarction renders suturing difficult, especially if extensive myocardial necrosis is present. Sutureless repair has gained popularity because of its simplicity and the lack of requirement for cardiopulmonary bypass. The use of several hemostasis patches has been reported in sutureless repair, including autologous pericardium and xenopericardium. Several articles reported the use of TachoSil for LVFWR. $(1,5)$ TachoSil is a ready-to-use collagen fleece that is coated with fibrin glue, containing fibrinogen, thrombin, and aprotinin, which has been used for hemostasis in cardiac surgeries. (1) To avoid re-rupture, we used three sheets of TachoSil in the present patient, to cover not only the necrotic area, but also an area of normal myocardium. This technique, to also cover healthy myocardium, has also been proposed by Raffa et al . previously. (5)

Okamura et al . reported 35 cases of sutureless repair for LVFWR. Re-rupture occurred in six patients within 1 week of the initial surgery, due to fragile myocardium at the time of surgery. (1) Left ventricular pressure overload should be avoided during this period, and there is a greater risk of hypertension if the patient is awake due to postoperative pain and delirium. Previous reports have suggested strict BP and heart rate control, bed rest, and the avoidance of agitation during the postoperative care of patients with LVFWR. (2) Although deep sedation has some disadvantages, including potential complications of ventilatorassociated pneumonia and weakening of the skeletal muscles, we considered that deep sedation for 2 weeks was acceptable to minimize the likelihood of re-rupture and ventricular aneurysm. Use of intra-aortic balloon pumping (IABP) is also recommended after surgery for circulatory support and reducing ventricular load. However, we did not use IABP in the present patient, because her hemodynamics was stable and the use of heparin may have predisposed this patient to a higher risk of bleeding from the necrotic myocardium.

Sutureless repair with TachoSil may be an effective method of treating blow-out type LVFWR. Deep sedation after surgery may improve the surgical outcomes by reducing ventricular load, thus preventing from re-rupture and formation of ventricular aneurysm .

\section{Author contributions}

Drafting of the manuscript: SK.

Critical revision of the manuscript: MS, DH, and AY.

Management of the manuscript writing: MS

\section{References}

1. Okamura H, Kimura N, Mieno M, Matsumoto H, Yuri K, Yamaguchi A. Sutureless repair for postinfarction left ventricular free wall rupture. The Journal of thoracic and cardiovascular surgery. 2019;158(3):771-7.

2. Matteucci M, Fina D, Jiritano F, Meani P, Blankesteijn WM, Raffa GM, et al. Treatment strategies for post-infarction left ventricular free-wall rupture. European heart journal Acute cardiovascular care. 2019;8(4):379-87.

3. Gong W, Shi H, Yan M, Yan Y, Wang X, Li S, et al. Clinical Manifestation, Timing Course, Precipitating Factors, and Protective Factors of Ventricular Free Wall Rupture Following ST-Segment Elevation Myocardial Infarction. International heart journal. 2020;61(4):651-7.

4. Matteucci M, Fina D, Jiritano F, Blankesteijn WM, Raffa GM, Kowalewski M, et al. Sutured and sutureless 
repair of postinfarction left ventricular free-wall rupture: a systematic review. European journal of cardiothoracic surgery : official journal of the European Association for Cardio-thoracic Surgery. 2019;56(5):840-8.

5. Raffa GM, Tarelli G, Patrini D, Settepani F. Sutureless repair for postinfarction cardiac rupture: a simple approach with a tissue-adhering patch. The Journal of thoracic and cardiovascular surgery. 2013;145(2):598-9.

Figure legends

Fig 1. Preoperative enhanced computed tomography image

The tear in the unenhanced myocardium in the posterior ventricular wall is visible.

\section{Fig 2. Postoperative coronary computed tomography images}

(A) Stenosis in the peripheral right coronary artery and hypoplasia of the circumflex coronary artery. (B) No ventricular aneurysm is present on $3 \mathrm{D}$ computed tomography.
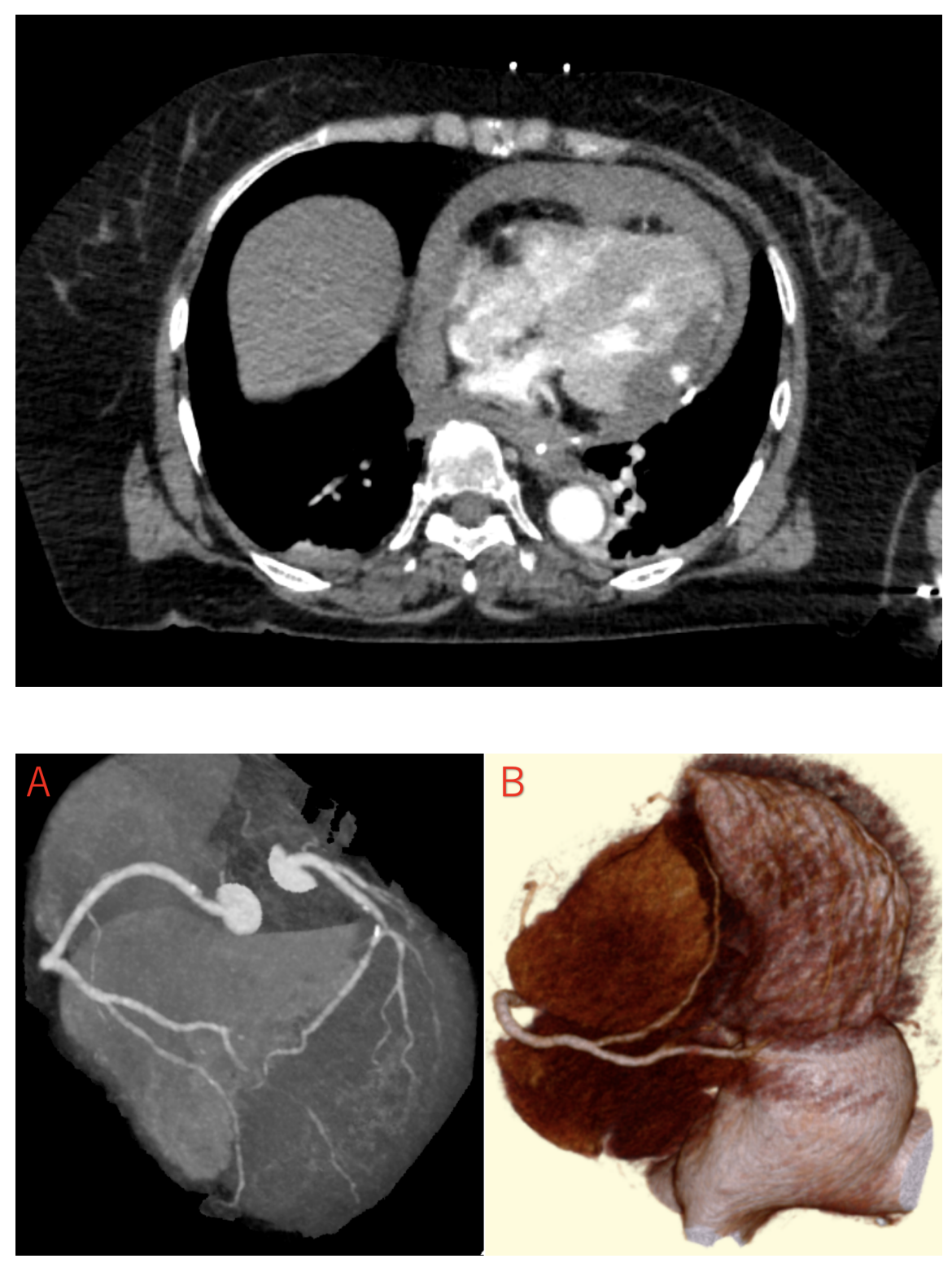